\title{
Effect of soil resistivity on the value of lightning induced voltage
}

\begin{abstract}
In this paper, lightning induced voltages on a distribution line associated with a lightning channel with reflection at the channel base were evaluated and compared with the corresponding values from a normal channel. Moreover, the effect of soil resistivity on the value of the lightning induced voltage and also lightning current and induced voltage behaviours versus soil resistivity changes were considered and the results discussed accordingly. The results showed that the reflected currents along channel can be strangely effective on the values of electromagnetic field and also lightning induced voltage. Therefore, in order to set proper protection on the distribution lines, the local information about electrical soil parameters should be taken into account.
\end{abstract}

Keyword: Lightning induced voltage; Ground reflection; Soil resistivity 